\title{
Alternatives to Remdesivir: Drug repurposing for inhibition of SARSCoV2 RNA dependent RNA polymerase
}

Kumar Sharp ( $\sim$ ksharp0016@gmail.com )

Government Medical College and Hospital, Jalgaon https://orcid.org/0000-0002-5034-8326

Research Article

Keywords: COVID-19, remdesivir, RdRp, saquinavir

Posted Date: May 7th, 2021

DOl: https://doi.org/10.21203/rs.3.rs-504456/v1

License: () (i) This work is licensed under a Creative Commons Attribution 4.0 International License.

Read Full License 


\section{Abstract}

Even after more than a year of the beginning of COVID-19 pandemic, a specific treatment for the disease has not been discovered. Vaccination programmes are being rolled out as the fastest pace possible but achievement of herd immunity will take time. ${ }^{[1]}$ Many drugs like favipiravir, remdesivir and tocilizumab are being used for the treatment of this disease but reports published by the World Health Organization and the New England Journal of Medicine shows that they do not produce any significant clinical results. In this study, by molecular docking a large set of drugs has been used to replace remdesivir in RdRp protein so that they can produce the same action and therefore provide suitable alternatives for clinical trials and emergency use. The drugs identified in the study are saquinavir, cefoperazone, gliquidone, nelfinavir, 5-methyltetrahyrofolate among various others.

\section{Introduction}

Even after more than a year of the beginning of COVID-19 pandemic, a specific treatment for the disease has not been discovered. Vaccination programmes are being rolled out as the fastest pace possible but achievement of herd immunity will take time. ${ }^{[1]}$ Many drugs like favipiravir, remdesivir and tocilizumab are being used for the treatment of this disease but reports published by the World Health Organization and the New England Journal of Medicine shows that they do not produce any significant clinical results. [2][3] The need for a specific treatment against COVID-19 is paramount. Drugs are chemical compounds which bind upon a specific protein to produce its effects. They can thus bind on other proteins too and can therefore provide repurposed alternative treatments. Remdesivir works by inhibiting the action of RNA dependent RNA polymerase RdRp of SARS-CoV2 which is necessary for viral RNA replication. ${ }^{[4]}$ In this study, by molecular docking a large set of drugs has been used to replace remdesivir in RdRp protein so that they can produce the same action and therefore provide suitable alternatives for clinical trials and emergency use.

\section{Methodology}

The general frame work of this study is based on one of my previous study with Dr. Shubhangi Dange ${ }^{[5]}$. It is depicted in the flowchart below: (please see figure 1)

\section{Step 1: Obtaining three-dimensional structures of receptor and ligands from the databases}

The three-dimensional structure of the receptor i.e. the RNA dependent RNA polymerase docked with remdesivir was obtained from the Protein Data Bank ${ }^{[6]}$ (PDB id: 7BV2). Ligand structures were obtained from Zinc15 database ${ }^{[7]}$. For this study, filters like "in-vivo" and "world" filters were used to choose the ligands. Repurposing an already approved drug is faster than creating any new novel compound. Therefore the "world" approved filter was used to sort only those compounds which have been approved 


\section{Step 2: Determining active site amino acid residues in the receptor}

W. Yin et al. stated in their study ${ }^{[8]}$ that "The complex structure reveals that the partial double-stranded RNA template is inserted into the central channel of the RdRp where Remdesivir is covalently incorporated into the primer strand at the first replicated base pair and terminates chain elongation." To find suitable alternatives to remdesivir, we would have to produce the same interactions that it produces with the other suitable compounds. Such type of attempt in-silico would have either produced unsatisfactory results or results which would prove to be useless in-vivo. Therefore, I figured out an alternate way to inhibit the action of RdRp. If we can inhibit the binding of the template RNA strand to RdRp then there would no production of the primer strand by replication. Even if it would occur, remdesivir could bind to it can further weaken or inhibit the process. So, the amino acids targeted in this study were those which bound to these RNA strands according to W. Yin et al. These amino acids are listed with their positions as follows:

1. $Y 915$

2. $Y 595$

3. F594

4. 5592

5. G590

6. A580

7. D684

8. A558

9. G683

10. G559

11. 5682

12. K500

13. N534

14. S501

15. Q541

16. N507

17. L854

18. 1847

19. $R 858$ 
20. $S 861$

21. D865

22. $\mathrm{R} 836$

23. $A 840$

24. Q815

25. $\mathrm{C} 813$

26. $S 814$

27. D761

28. $\$ 759$

29. D760

\section{Step 3: Preparation of receptor and ligand for docking}

The receptor i.e. the RdRp protein was first processed in Drug Discovery studio ${ }^{\left[{ }^{[9}\right.}$ by removal of heterogenous atoms, water molecules, prime and template RNA strands and remdesivir. This gave us the clean RdRp molecule. Using PyRx docking software ${ }^{[10]}$, it was converted automatically into an Autodock macromolecule. Around 750 drug molecules were loaded into PyRx using Open Babel plugin ${ }^{[11]}$ which were then converted into Autodock ligands by minimization of their energies, addition of hydrogen atoms and addition of partial charges.

\section{Step 4: Docking of ligands to the receptor within restricted search space of target amino acids}

Using Autodock Vina [12], ligands were docked into the restricted search space containing the target amino acids in step 2. The search parameters set were as follows:

center_x $=81.0674935587$

center_y $=90.6082924987$

center_z $=112.717699441$

size_x $=27.5120174204$

size_y $=40.1660106465$

size_z $=39.5156154165$

The docking was conducted with maximum exhaustiveness of 4 modes. The study was conducted on a Windows 10 64-bit operating system which took about 8 hours to complete. 


\section{Step 5: Sorting of result on basis of binding affinity, interactions, inclusion and exclusion criteria.}

The results obtained were filtered to include only the best mode of each ligand which had RMSD value of

0 . They were then sorted from the highest to lowest order of binding affinity. The names of the ligands were derived from the Zinc15 database.

\section{Step 6: Final potential drug candidates obtained.}

Literature search about any possible association of these drugs to COVID-19 was done. Top 20 drugs were chosen and were included in this study.

\section{Results}

The final 20 drugs obtained from the study are displayed below in decreasing order of binding affinity. 


\begin{tabular}{|lll|}
\hline Ligand & $\begin{array}{l}\text { Binding Affinity } \\
\text { (kcal/mol) }\end{array}$ & $\begin{array}{l}\text { Name (as per Zinc15 } \\
\text { database) }\end{array}$ \\
\hline rdrp_7bv2_clean_ZINC000003914596 & -8.6 & Saquinavir \\
\hline rdrp_7bv2_clean_ZINC000003830431 & -8.6 & Cefoperazone \\
\hline rdrp_7bv2_clean_ZINC000001482077 & -8.6 & Gliquidone \\
\hline rdrp_7bv2_clean_ZINC000003831231 & -8.5 & Novobiocin \\
\hline rdrp_7bv2_clean_ZINC000003927822 & -8.3 & Lurasidone \\
\hline rdrp_7bv2_clean_ZINC000000537877 & -8.3 & Ketanserin \\
\hline rdrp_7bv2_clean_ZINC000001481956 & -8.1 & Paliperidone \\
\hline rdrp_7bv2_clean_ZINC000001530886 & -8 & Telmisartan \\
\hline rdrp_7bv2_clean_ZINC000000538337 & -7.9 & Sertindole \\
\hline rdrp_7bv2_clean_ZINC000001542113 & -7.9 & Vilazodone \\
\hline rdrp_7bv2_clean_ZINC000003914813 & -7.8 & Tudca \\
\hline rdrp_7bv2_clean_ZINC000001542146 & -7.7 & Pranlukast \\
\hline rdrp_7bv2_clean_ZINC000003872994 & -7.7 & Nizoral \\
\hline rdrp_7bv2_clean_ZINC000001543181 & -7.7 & Ibutamoren \\
\hline rdrp_7bv2_clean_ZINC000003833846 & -7.6 & Nelfinavir \\
\hline rdrp_7bv2_clean_ZINC000001489478 & -7.6 & Sitagliptin \\
\hline rdrp_7bv2_clean_ZINC000001481831 & -7.5 & Sitaxentan \\
\hline rdrp_7bv2_clean_ZINC000002005305 & -7.5 & 5-methyltetrahydrofolate \\
\hline rdrp_7bv2_clean_ZINC000000586239 & -7.5 & Deferasirox/exjade \\
\hline rdrp_7bv2_clean_ZINC000001481815 & -7.5 & \\
\hline
\end{tabular}

The drug interactions of all the molecules were studied in Drug Discover studio and the number of interactions which matched our target amino acids were noted.

\section{Discussion}

Saquinavir has been found to have inhibitory action on SARS-CoV2 spike glycoprotein and 3CL main protease ${ }^{[13]}$. Cefoperazone was included in the study because of its widespread use and availability as well as the high number of target amino acids it interacted with. However, no study linking it directly to inhibition of SARS-CoV2 was found. Gliquidone was found to have inhibitory effect on 3CL main protease as well as RdRp ${ }^{[14]}{ }^{[15]}$. Novobiocin was identified as an inhibitor of heat shock protein 90 which 
allowed virus to control infected cells ${ }^{[16]}$. Lurasidone was found as the inhibitor of $3 \mathrm{CL}$ main protease in an in-silico study ${ }^{[17]}$. Ketanserin is being studied as aa potential additive drug to improve V/Q mismatch in COVID-19 ${ }^{[18]}$. No study linking paliperidone to SARS-CoV2 inhibition was found but it was included in the study due to the number of interactions as well as binding affinity. Telmisartan was found to demonstrate anti-inflammatory effect and improved morbidity in hospitalized patients infected with SARS-CoV2 ${ }^{[19]}$. Sertindole had inhibitory effect on main protease ${ }^{[20]}$. No study linking vilazodone to SARS-CoV2 inhibition was found but it was included in the study due to the number of interactions as well as binding affinity. Tudca/ ursodeoxycholic acid proven safety profiles that can reduce inflammation and prevent cell death to reduce morbidity and mortality in COVID-19 [21]. Pranlukast can improve COVID19 prognosis ${ }^{[22]}{ }^{[23]}$. No study linking nizoral/ketoconazole to SARS-CoV2 inhibition was found but can reduce host vulnerability to COVID-19 [24]. Ibutamoren had inhibitory effect on main protease ${ }^{[25]}$. Nelfinavir has been found to inhibit replication of SARS-CoV2 invitro ${ }^{[26]}$. Sitagliptin administration was associated with reduced mortality in patients with type 2 diabetes and COVID-19 [27]. Sitaxentan, an endothelin receptor blocker, can improve prognosis of COVID-19 ${ }^{[28]}$. 5-methyltetrahydrofolate is a potential treatment for pulmonary hypertension associated with COVID-19 pneumonia ${ }^{[29]}$. Levocabastin can be used against SARS-CoV2 spike proteins as a nasal spray ${ }^{[30]}$. Deferasirox can be used as an iron chelator to reduce or hamper virus survival ${ }^{[31]}$.

\section{Conclusion}

The drugs obtained above have also been identified in other in-silico studies as potential inhibitors of SARS-CoV2 by different mechanisms. They must be tried in-vitro because of the limitations of in-silico docking ${ }^{[32]}$. Alternatives and/or additives to remdesivir are important to help us come out of this crisis as soon as possible. These drugs can be expected to work on various proteins or mechanisms at the same time and provide quicker recovery of the patient.

\section{Declarations}

\section{Source of funding: Nil}

Ethical approval: Not required

Conflict of interest: The author hereby declares no conflict of interests.

\section{References}

1. How vaccine nationalism may delay global herd immunity [Internet]. Medicalnewstoday.com. 2021 [cited 4 May 2021]. Available from: https://www.medicalnewstoday.com/articles/herd-immunitymay-take-4-6-years-due-to-vaccine-nationalism 
2. WHO Solidarity Trial Consortium. Repurposed antiviral drugs for COVID-19-interim WHO SOLIDARITY trial results. New England journal of medicine. 2021 Feb 11;384(6):497-511.

3. Rosas IO, Bräu N, Waters M, Go RC, Hunter BD, Bhagani S, Skiest D, Aziz MS, Cooper N, Douglas IS, Savic S. Tocilizumab in hospitalized patients with severe Covid-19 pneumonia. New England Journal of Medicine. 2021 Apr 22;384(16):1503-16.

4. Al-Tawfiq JA, Al-Homoud AH, Memish ZA. Remdesivir as a possible therapeutic option for the COVID19. Travel medicine and infectious disease. 2020 Mar 5.

5. Sharp, K., Dange, S. In-silico FDA-approved drug repurposing to find the possible treatment of Coronavirus Disease-19 (COVID-19). Indian J. Biotech. Pharm. Res. 2020; 8(2): 1-10

6. Bank PD. Protein data bank. Nature New Biol. 1971; 233:223.

7. Sterling T, Irwin JJ. ZINC 15-ligand discovery for everyone. Journal of chemical information and modelling. 2015 Nov 23;55(11):2324-37.

8. Yin W, Mao C, Luan X, Shen DD, Shen Q, Su H, Wang X, Zhou F, Zhao W, Gao M, Chang S. Structural basis for inhibition of the RNA-dependent RNA polymerase from SARS-CoV-2 by remdesivir. Science. 2020 Jun 26;368(6498):1499-504.

9. Ref. Dassault Systèmes BIOVIA, Discovery Studio Modeling Environment, Release 2017, San Diego: Dassault Systèmes, 2016.

10. Dallakyan S, Olson AJ. Small-molecule library screening by docking with PyRx. InChemical biology 2015 (pp. 243-250). Humana Press, New York, NY.

11. O'Boyle NM, Banck M, James CA, Morley C, Vandermeersch T, Hutchison GR. Open Babel: An open chemical toolbox. Journal of cheminformatics. 2011 Dec;3(1):33.

12. Trott $\mathrm{O}$, Olson AJ. AutoDock Vina: improving the speed and accuracy of docking with a new scoring function, efficient optimization, and multithreading. Journal of computational chemistry. 2010 Jan 30;31(2):455-61.

13. Hall Jr DC, Ji HF. A search for medications to treat COVID-19 via in silico molecular docking models of the SARS-CoV-2 spike glycoprotein and 3CL protease. Travel medicine and infectious disease. 2020 May 1; 35:101646.

14. Qu H, Zheng Y, Wang Y, Li H, Liu X, Xiong X, Zhang L, Gu J, Yang G, Zhu Z, Zheng H. The potential effects of clinical antidiabetic agents on SARS-CoV-2. Journal of diabetes. 2021 Mar;13(3):243-52.

15. Khater S, Dasgupta N, Das G. Combining SARS-CoV-2 proofreading exonuclease and RNA-dependent RNA polymerase inhibitors as a strategy to combat COVID-19: a high-throughput in silico screen.

16. Sultan I, Howard S, Tbakhi A. Drug repositioning suggests a role for the heat shock protein 90 inhibitor geldanamycin in treating COVID-19 infection.

17. Elmezayen AD, Al-Obaidi A, Şahin AT, Yelekçi K. Drug repurposing for coronavirus (COVID-19): in silico screening of known drugs against coronavirus $3 \mathrm{CL}$ hydrolase and protease enzymes. Journal of Biomolecular Structure and Dynamics. 2020 Apr 24:1-3. 
18. Kuindersma M, Spronk PE. Ketanserin as potential additive drug to improve V/Q mismatch in COVID19? Critical Care. 2020 Dec;24(1):1-2.

19. Duarte M, Pelorosso FG, Nicolosi L, Salgado MV, Vetulli H, Aquieri A, Azzato F, Basconcel M, Castro M, Coyle J, Davolos I. Telmisartan for treatment of Covid-19 patients: an open randomized clinical trial. Preliminary report. medRxiv. 2020 Jan 1.

20. Vatansever EC, Yang K, Kratch KC, Drelich A, Cho CC, Mellot DM, Xu S, Tseng CT, Liu WR. Targeting the SARS-CoV-2 main protease to repurpose drugs for COVID-19. BioRxiv. 2020 Jan 1.

21. Subramanian S, Iles T, Ikramuddin S, Steer CJ. Merit of an ursodeoxycholic acid clinical trial in COVID-19 patients. Vaccines. 2020 Jun;8(2):320.

22. Rao A, Saipradeep VG, Joseph T, Kotte S, Sivadasan N, Srinivasan R. Text and network-mining for covid-19 intervention studies.

23. Barré J, Sabatier JM, Annweiler C. Montelukast Drug May Improve COVID-19 Prognosis: A Review of Evidence. Frontiers in Pharmacology. 2020 Sep 4; 11:1344.

24. Barbot M, Ceccato F, Scaroni C. Consideration on TMPRSS2 and the risk of COVID-19 infection in Cushing's syndrome. Endocrine. 2020 Aug;69(2):235-6.

25. Durdagi S, Aksoydan B, Dogan B, Sahin K, Shahraki A, Birgül-jyison N. Screening of clinically approved and investigation drugs as potential inhibitors of SARS-CoV-2 main protease and spike receptor-binding domain bound with ACE2 COVID19 target proteins: a virtual drug repurposing study.

26. Yamamoto N, Matsuyama S, Hoshino T, Yamamoto N. Nelfinavir inhibits replication of severe acute respiratory syndrome coronavirus 2 in vitro. BioRxiv. 2020 Jan 1.

27. Solerte SB, D’Addio F, Trevisan R, Lovati E, Rossi A, Pastore I, Dell'Acqua M, Ippolito E, Scaranna C, Bellante R, Galliani S. Sitagliptin treatment at the time of hospitalization was associated with reduced mortality in patients with type 2 diabetes and COVID-19: a multicenter, case-control, retrospective, observational study. Diabetes Care. 2020 Dec 1;43(12):2999-3006.

28. Sanghavi DK, Titus A, Caulfield TR, Freeman WD. Endotheliitis, endothelin, and endothelin receptor blockers in COVID-19. Medical Hypotheses. 2021 May 1; 150:110564.

29. Wiltshire E, Peña AS, MacKenzie K, Shaw G, Couper J. High dose folic acid is a potential treatment for pulmonary hypertension, including when associated with COVID-19 pneumonia. Medical hypotheses. 2020 Oct 1; 143:110142.

30. Khan MF, Ansari WA, Ahamad T, Khan MA, Khan ZA, Sarfraz A, Khan MA. Bioactive Agents Contained in Different Nasal Sprays May Defeat SARS-Cov-2: A Repurposing and In-Silico Approach.

31. Liu W, Zhang S, Nekhai S, Liu S. Depriving iron supply to the virus represents a promising adjuvant therapeutic against viral survival. Current clinical microbiology reports. 2020 Jun;7(2):13-9.

\section{Figures}




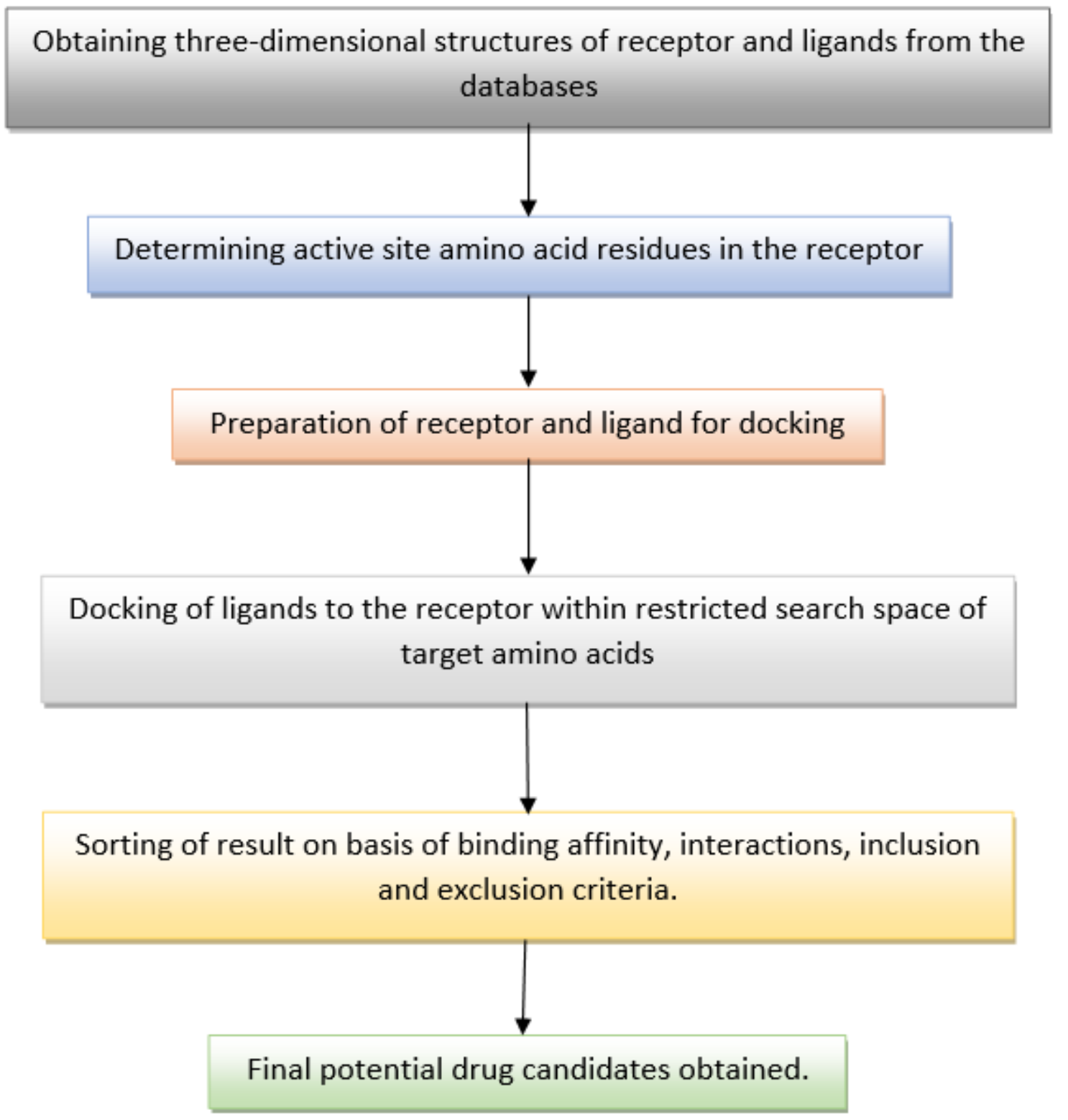

\section{Figure 1}

General summary of the study methodology followed. 

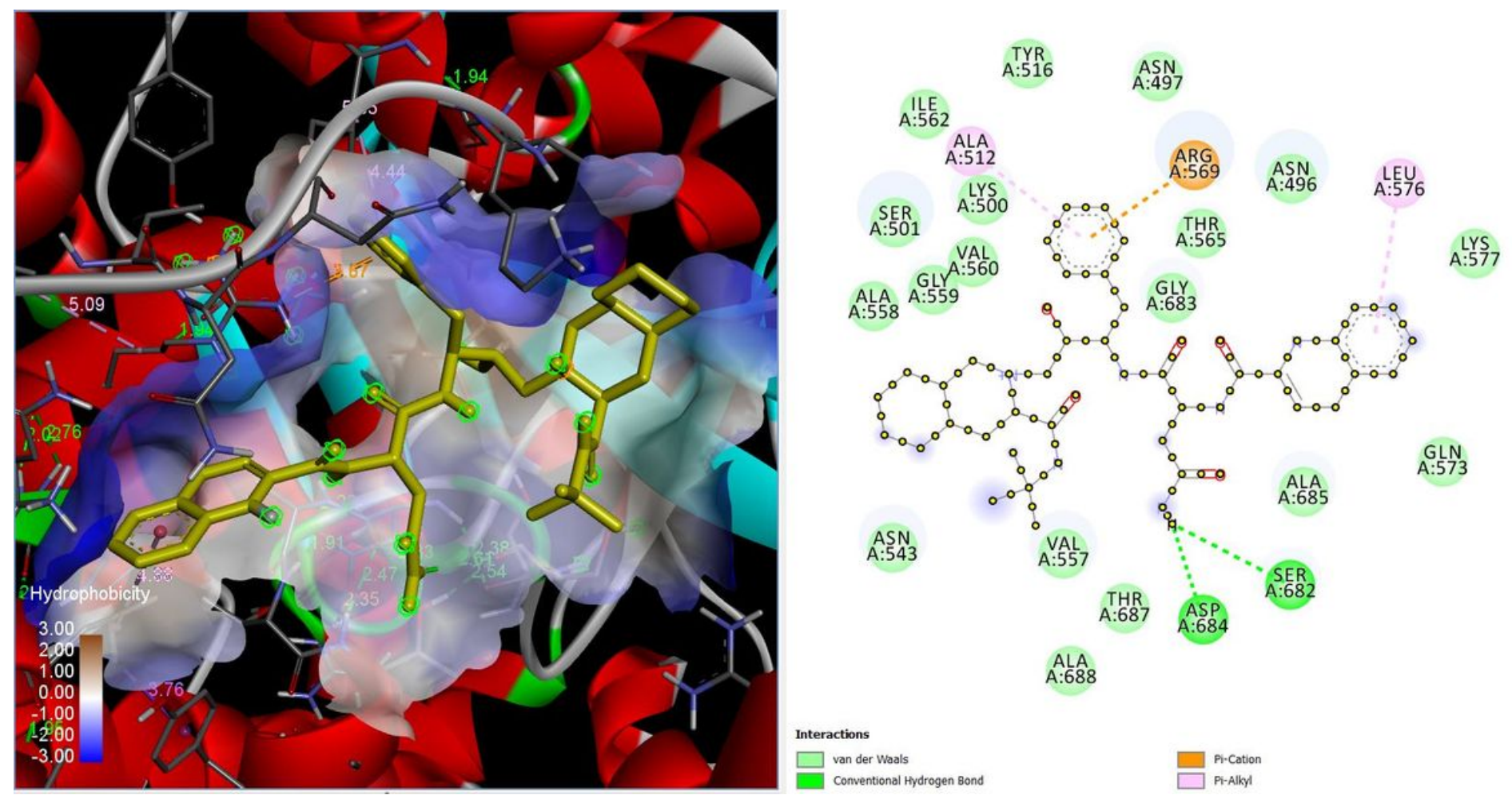

Figure 2

Saquinavir in its docking space in RdRp visualized using Drug Discover Studio. 7 target amino acids were interacted by it.
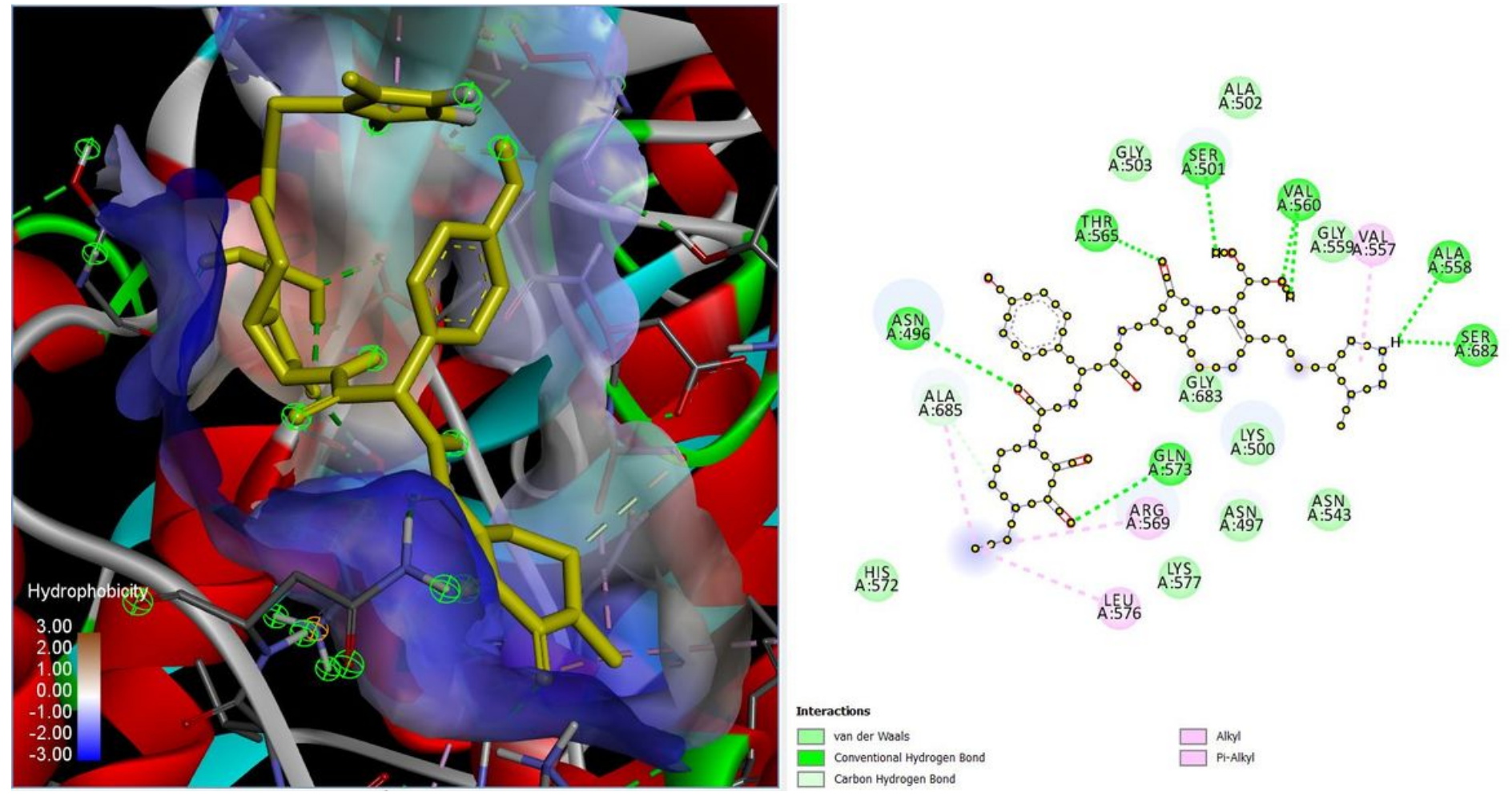

Figure 3 
Cefoperazone in its docking space in RdRp visualized using Drug Discover Studio. 6 target amino acids were interacted by it.

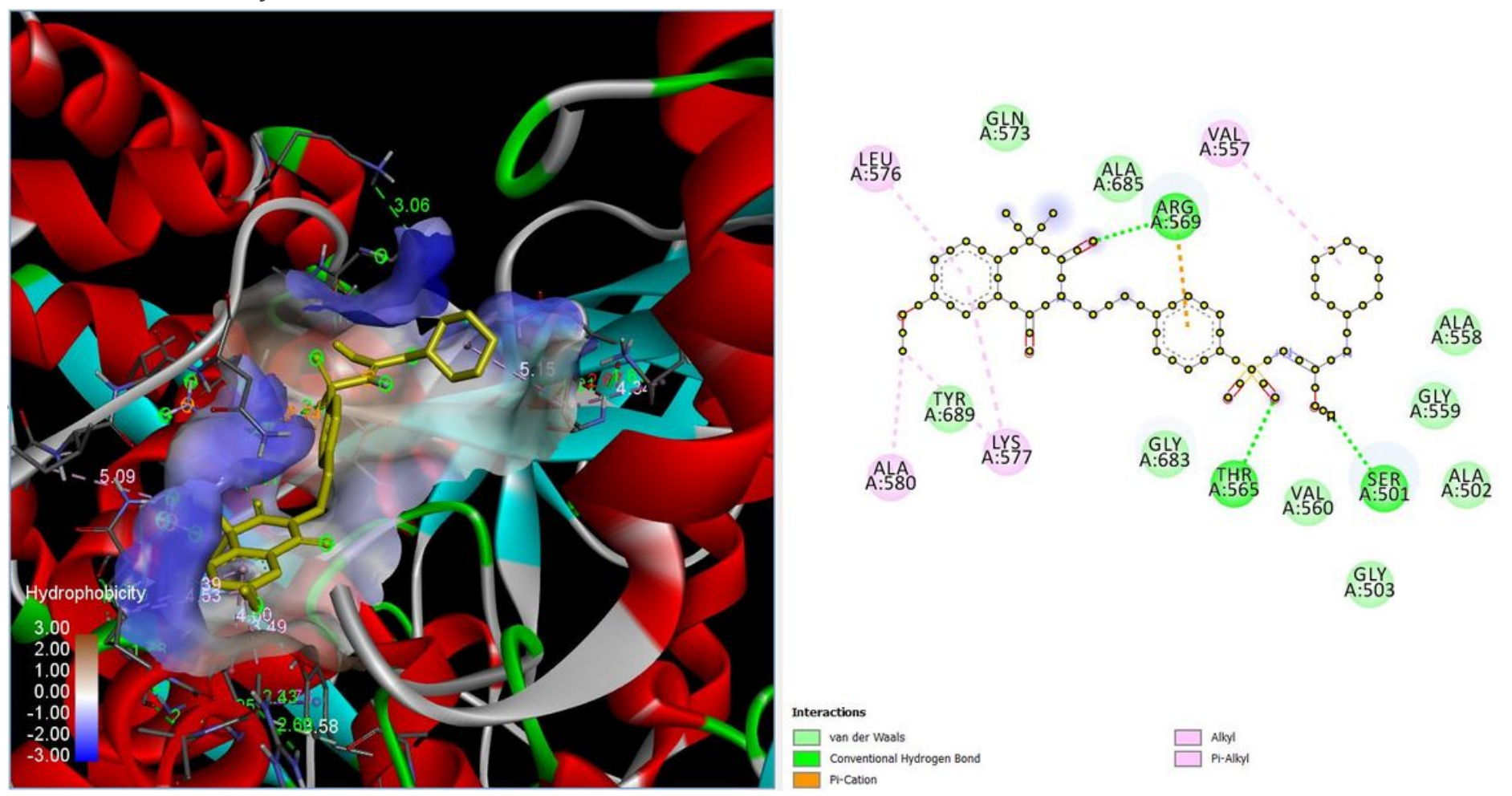

\section{Figure 4}

Gliquidone in its docking space in RdRp visualized using Drug Discover Studio. 5 target amino acids were interacted by it.
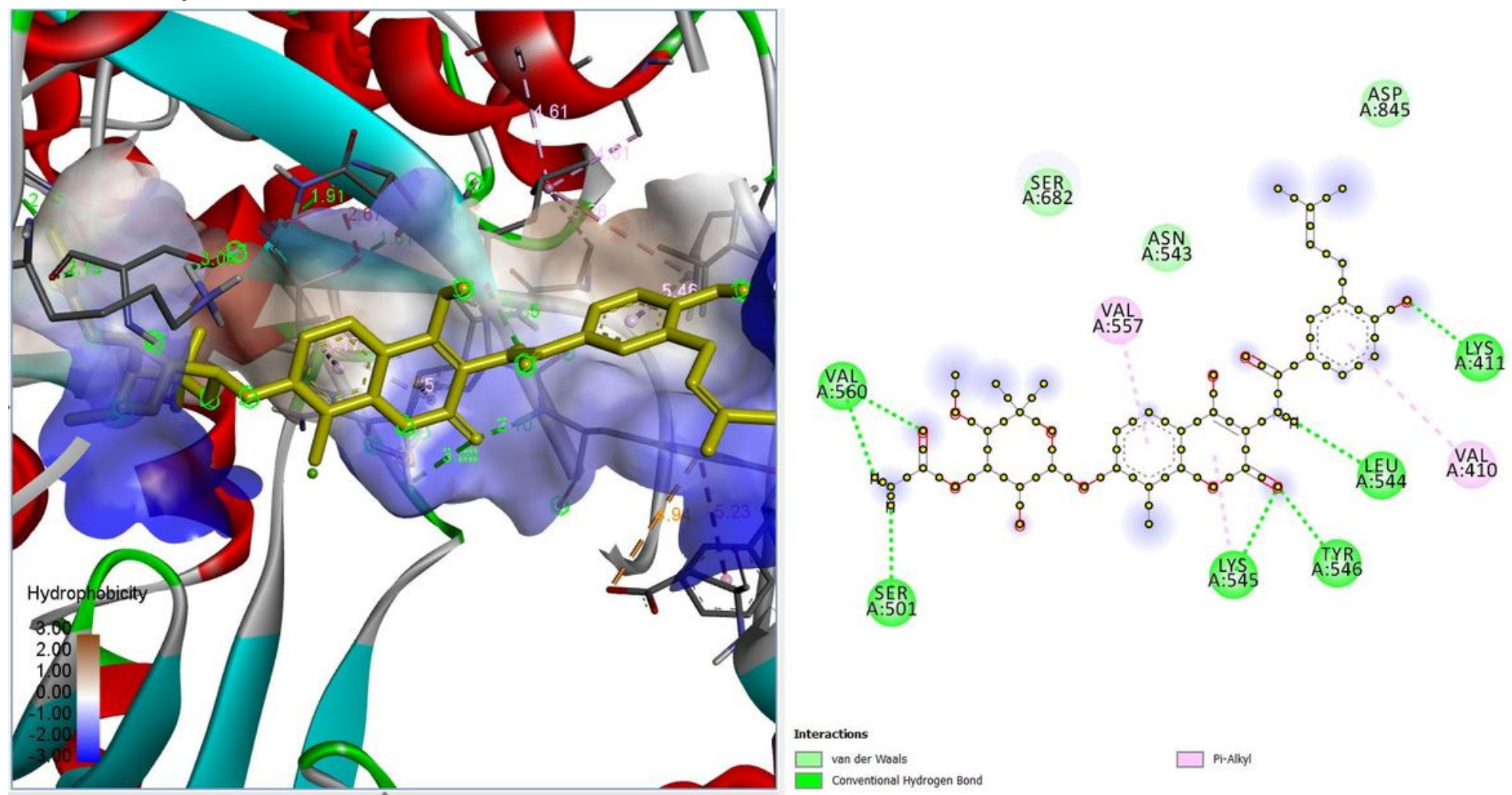

Figure 5 
Novobiocin in its docking space in RdRp visualized using Drug Discover Studio. 2 target amino acids were interacted by it.
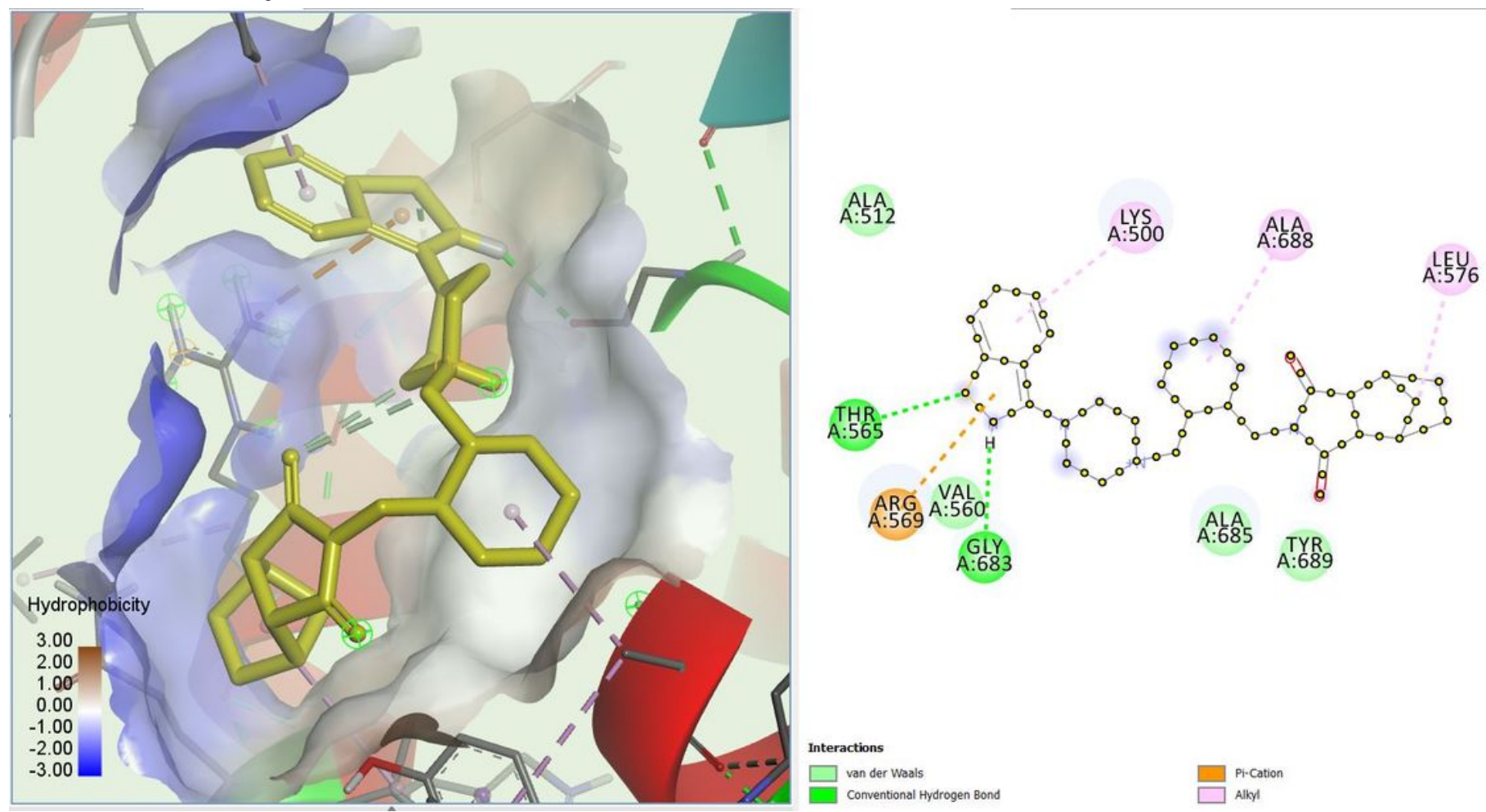

Figure 6

Lurasidone in its docking space in RdRp visualized using Drug Discover Studio. 2 target amino acids were interacted by it.
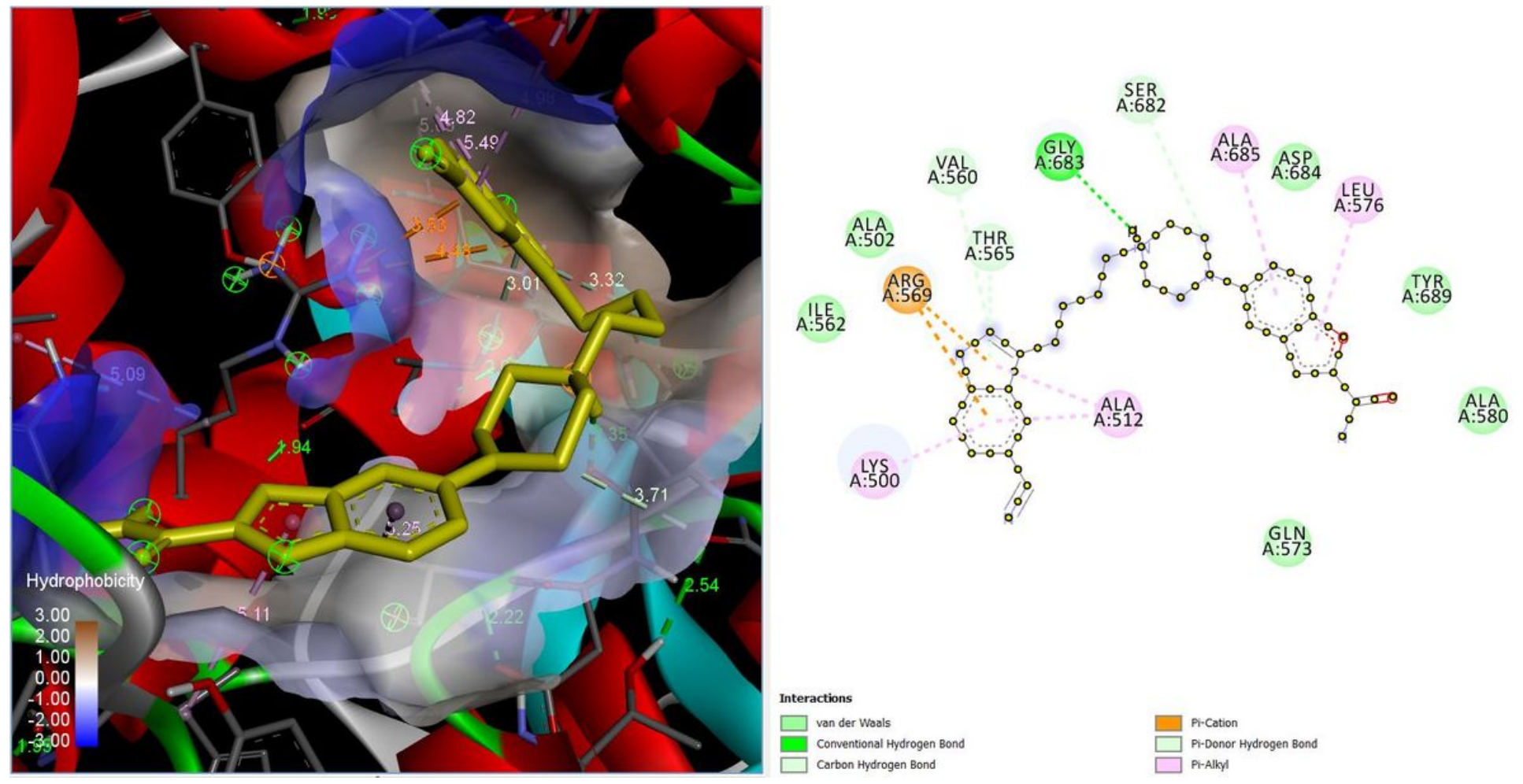

Figure 7 
Ketanserin in its docking space in RdRp visualized using Drug Discover Studio. 0 target amino acids were interacted by it.
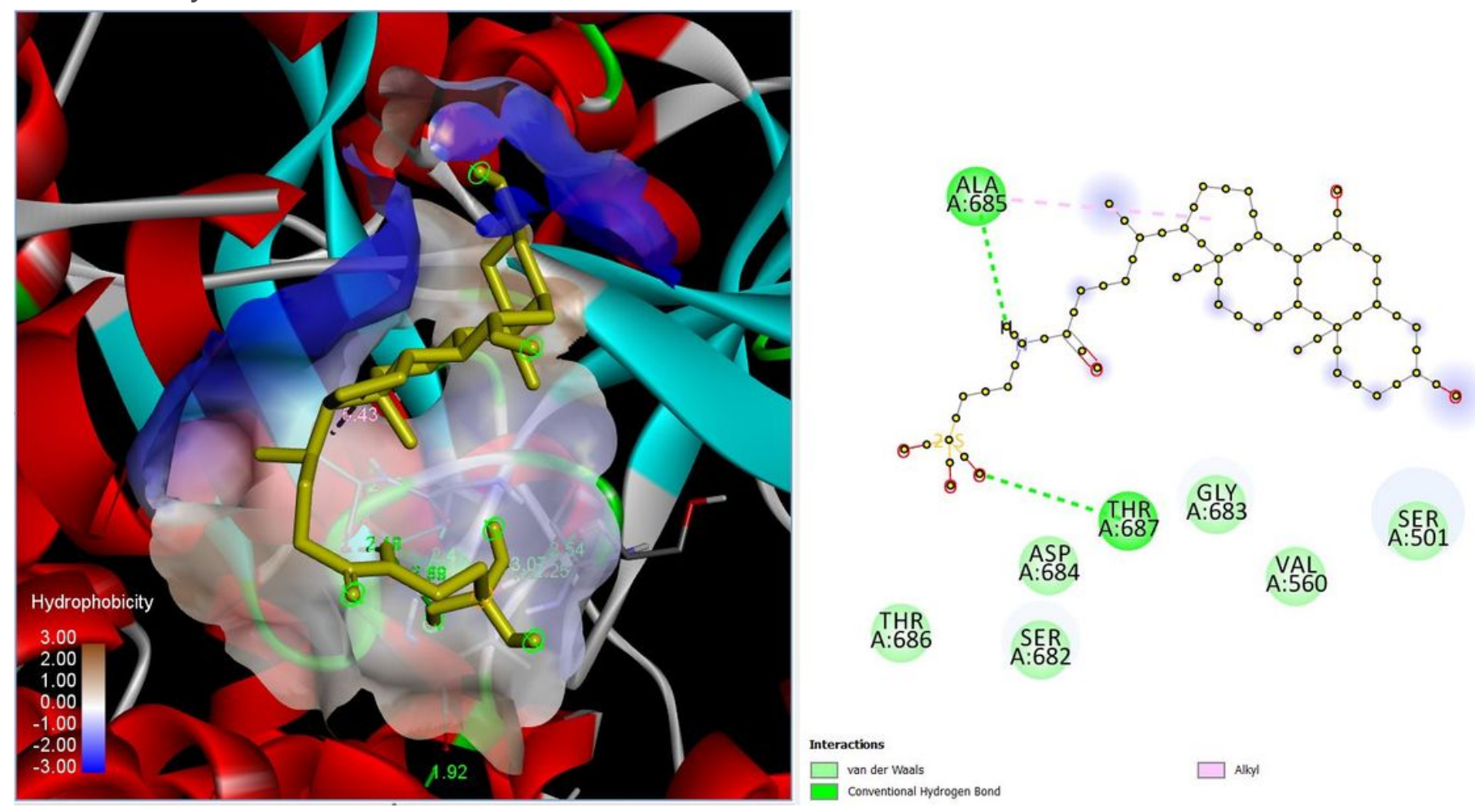

Figure 8

Palperidone in its docking space in RdRp visualized using Drug Discover Studio. 4 target amino acids were interacted by it.
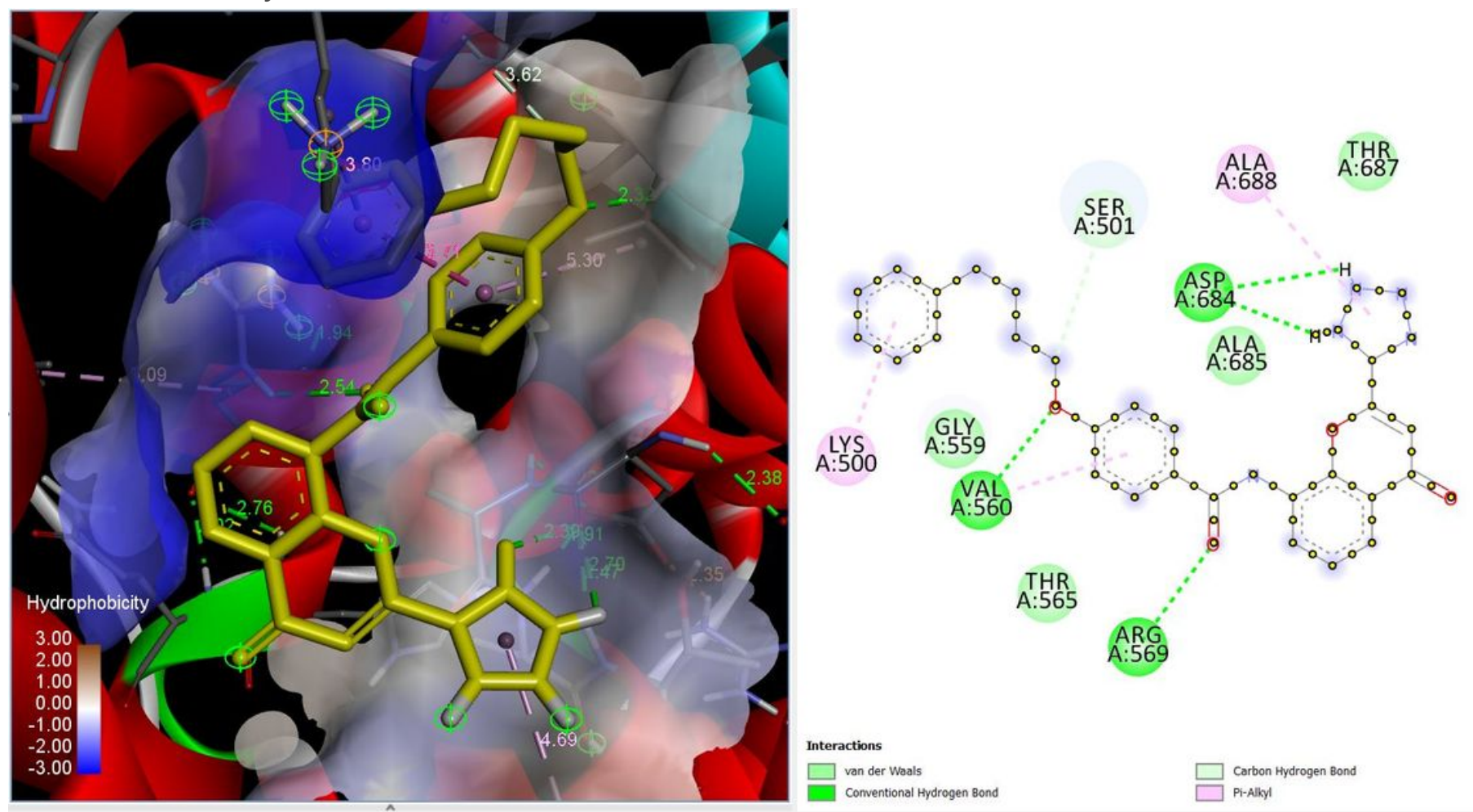

Figure 9 
Telmisartan in its docking space in RdRp visualized using Drug Discover Studio. 3 target amino acids were interacted by it.
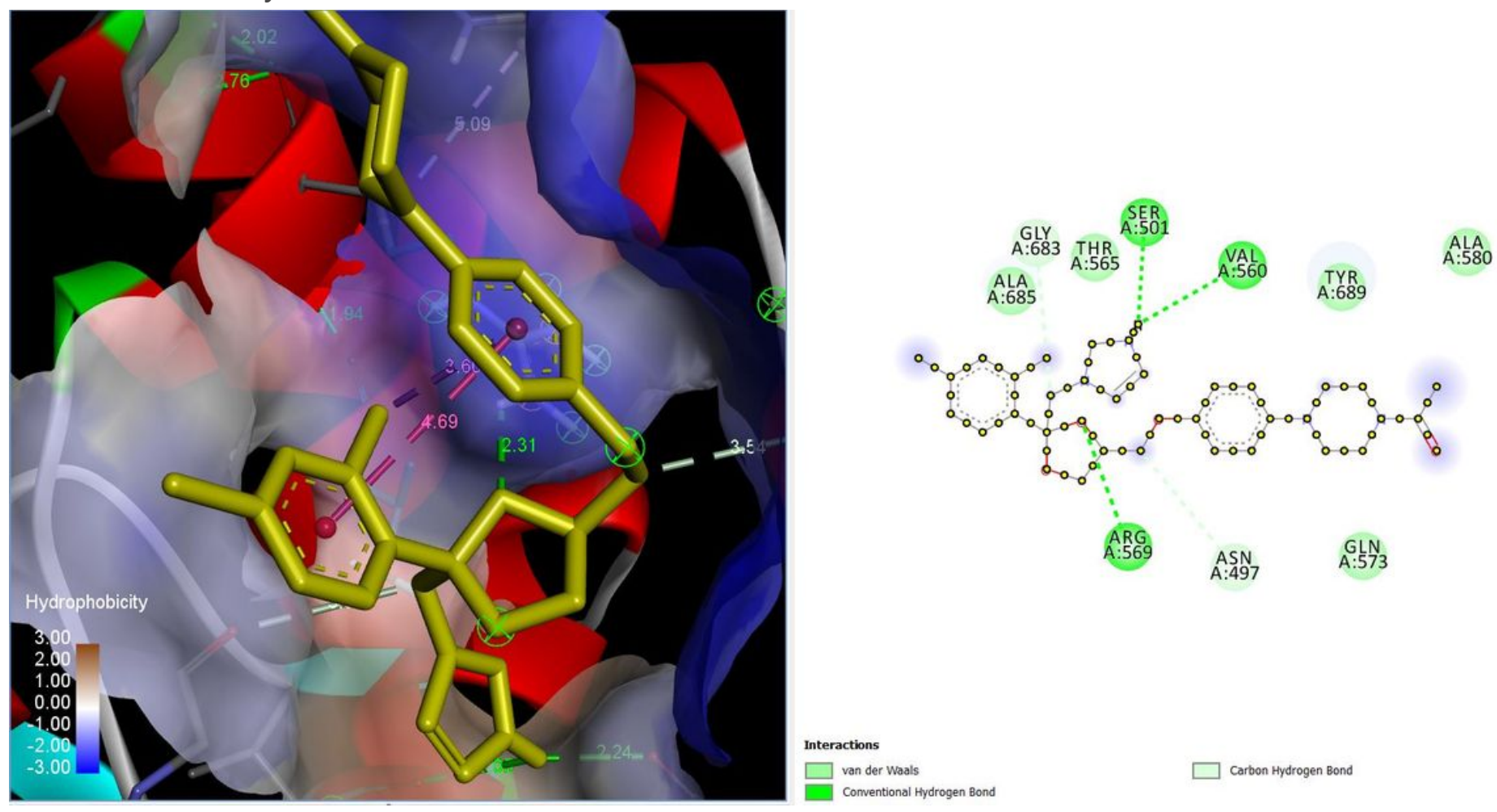

Figure 10

Sertindole in its docking space in RdRp visualized using Drug Discover Studio. 6 target amino acids were interacted by it.
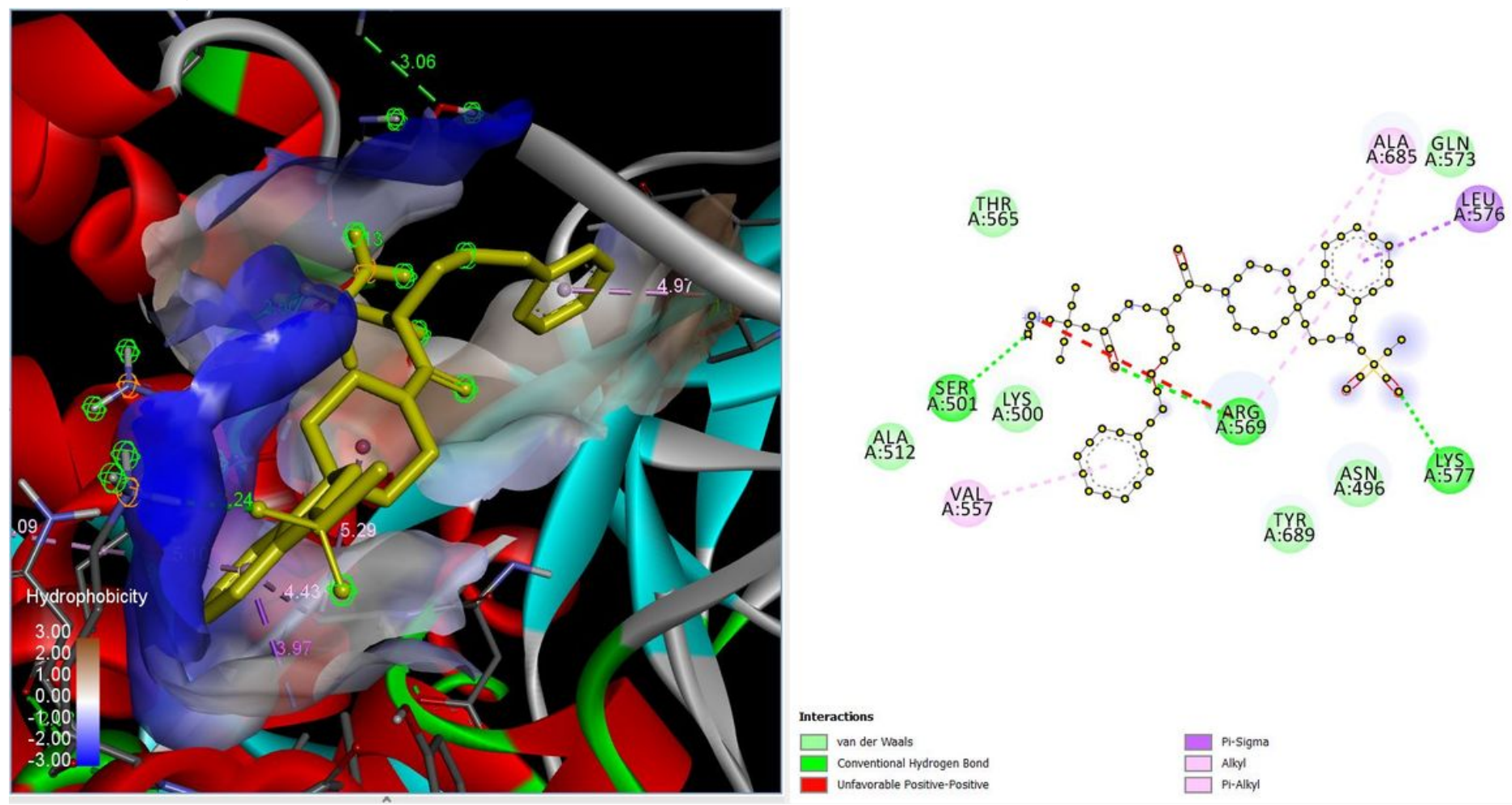

Figure 11 
Vilazodone in its docking space in RdRp visualized using Drug Discover Studio. 5 target amino acids were interacted by it.
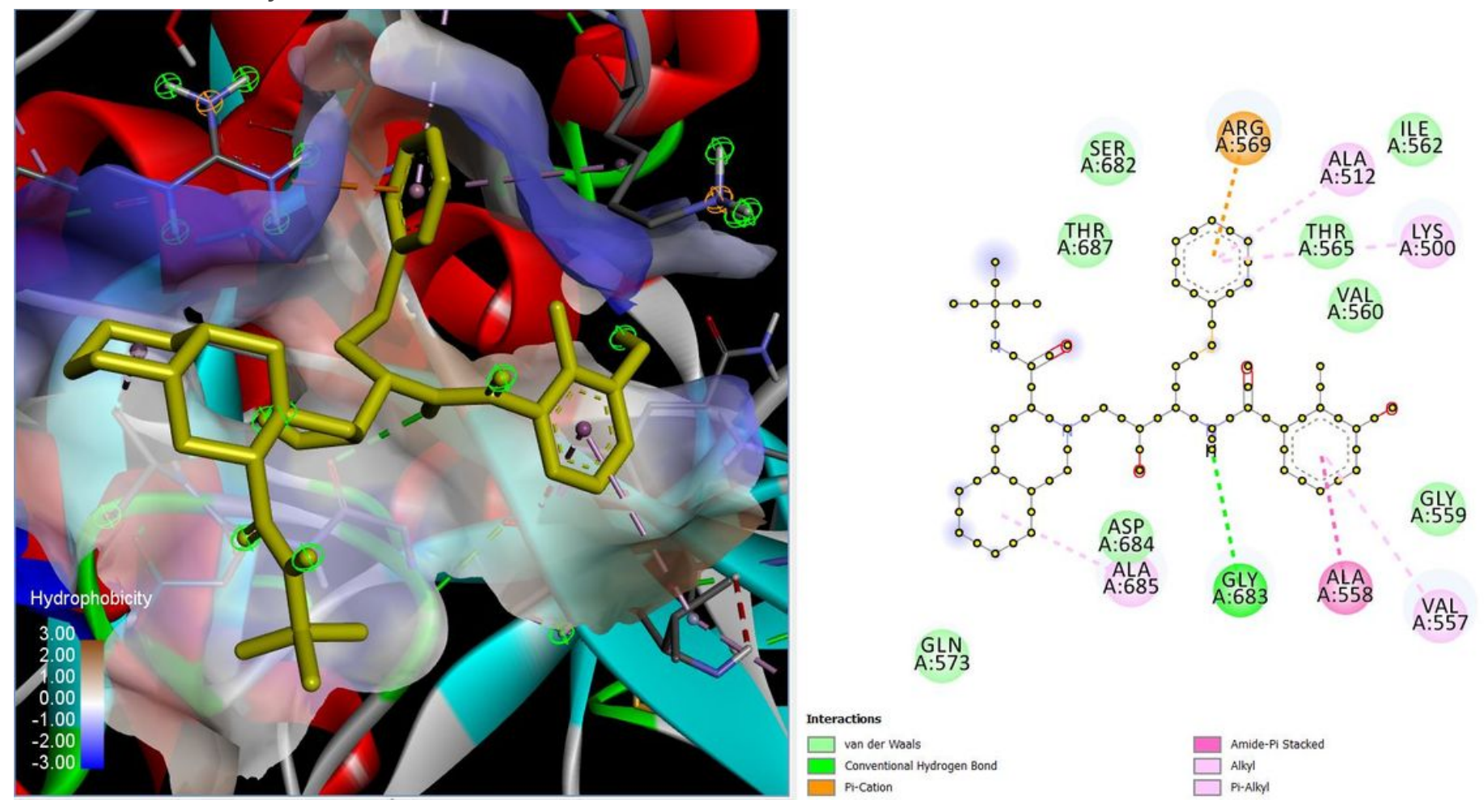

Figure 12

Tudca in its docking space in RdRp visualized using Drug Discover Studio. 4 target amino acids were interacted by it.
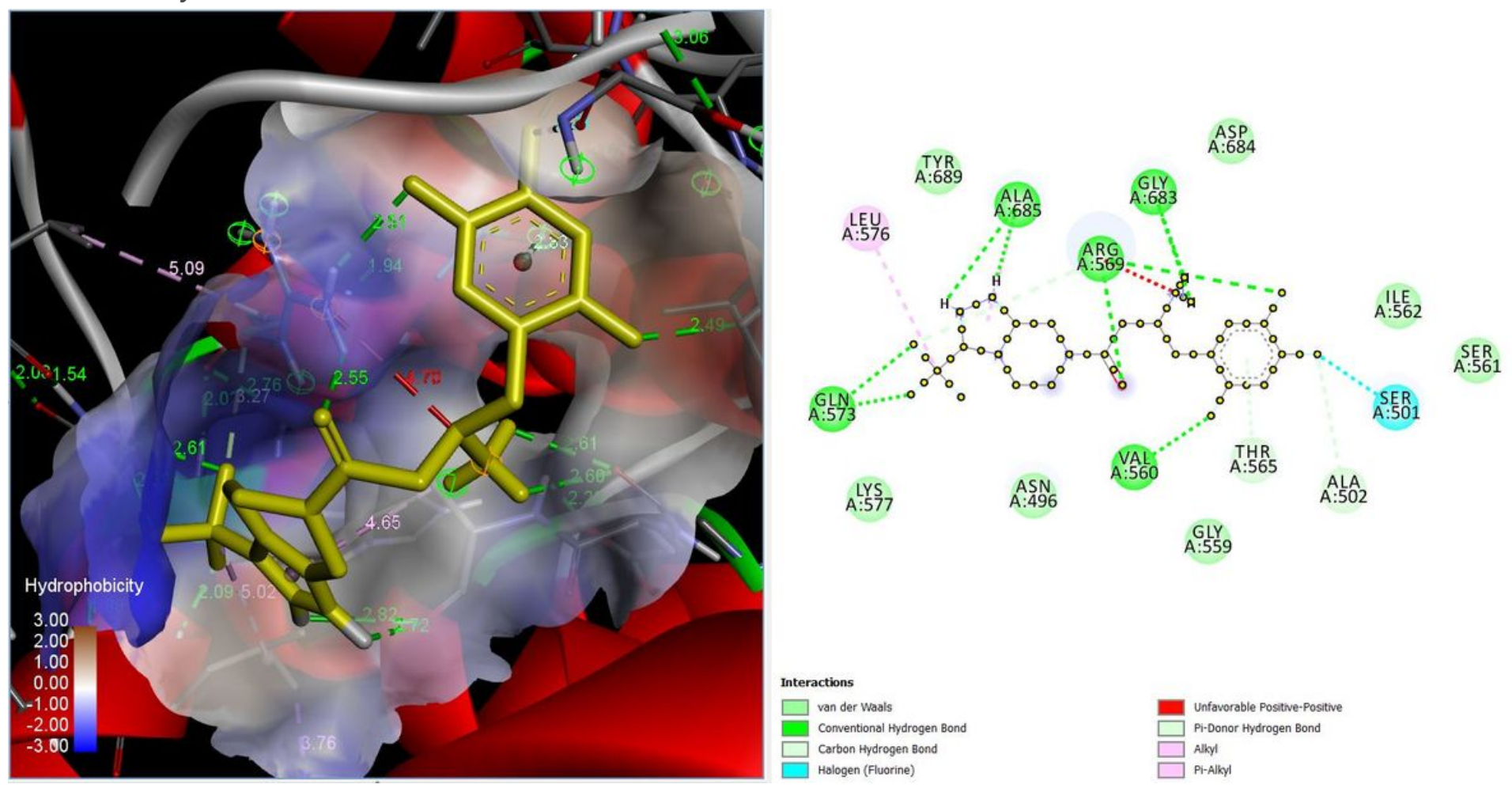

Figure 13 
Pranlukast in its docking space in RdRp visualized using Drug Discover Studio. 4 target amino acids were interacted by it.
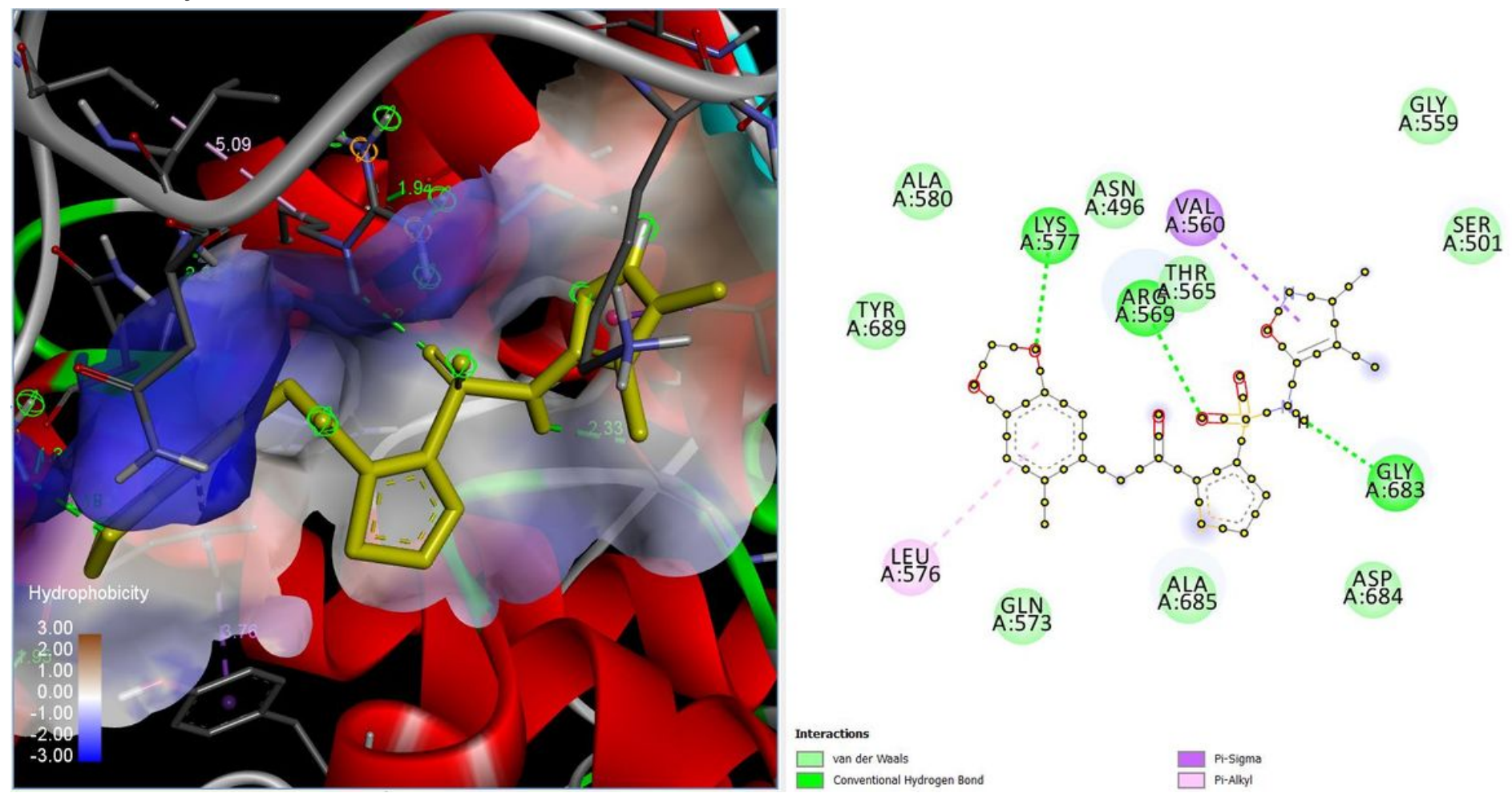

Figure 14

Nizoral in its docking space in RdRp visualized using Drug Discover Studio. 3 target amino acids were interacted by it.
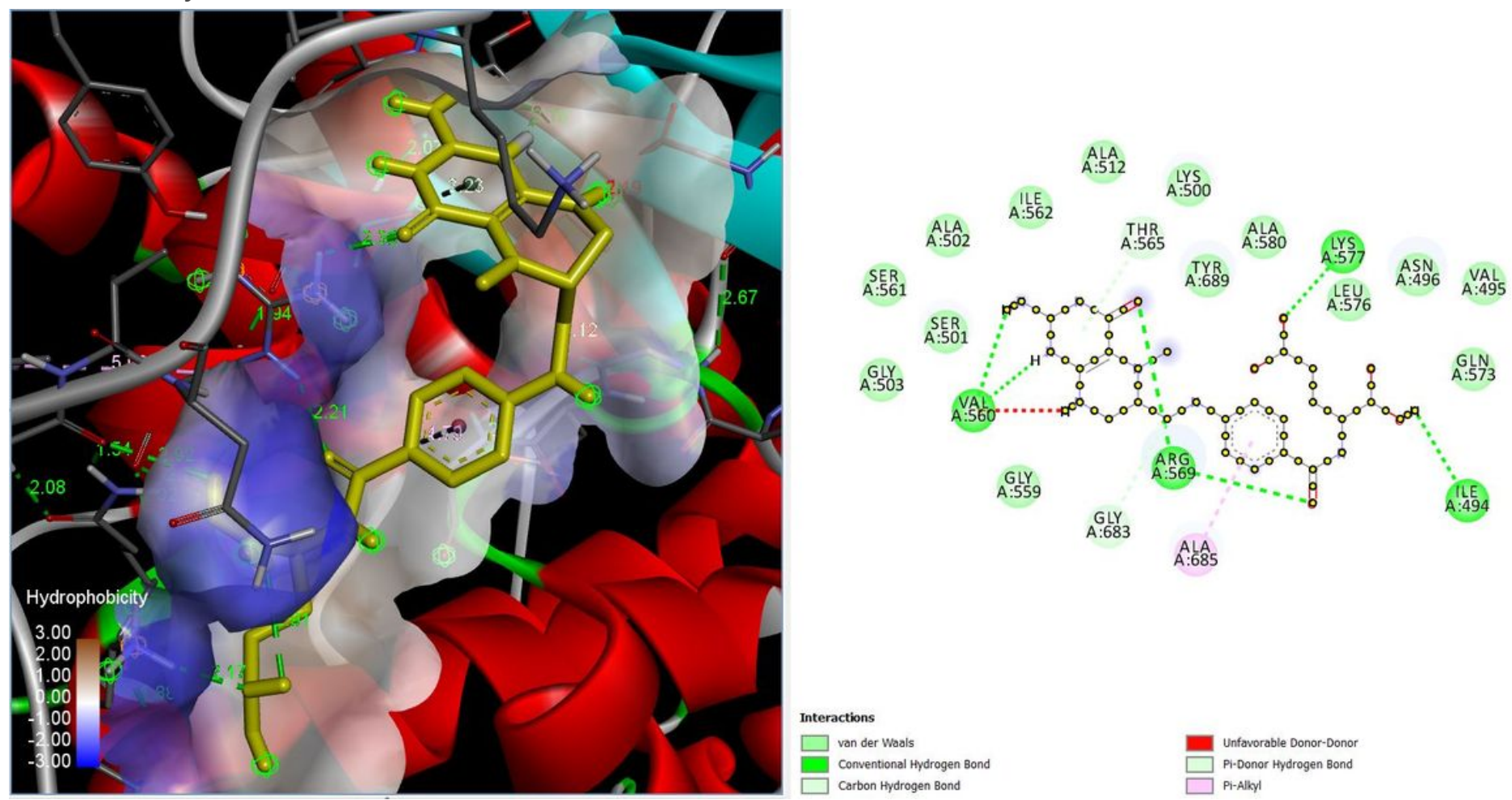

Figure 15 
Ibutamoren in its docking space in RdRp visualized using Drug Discover Studio. 2 target amino acids were interacted by it.
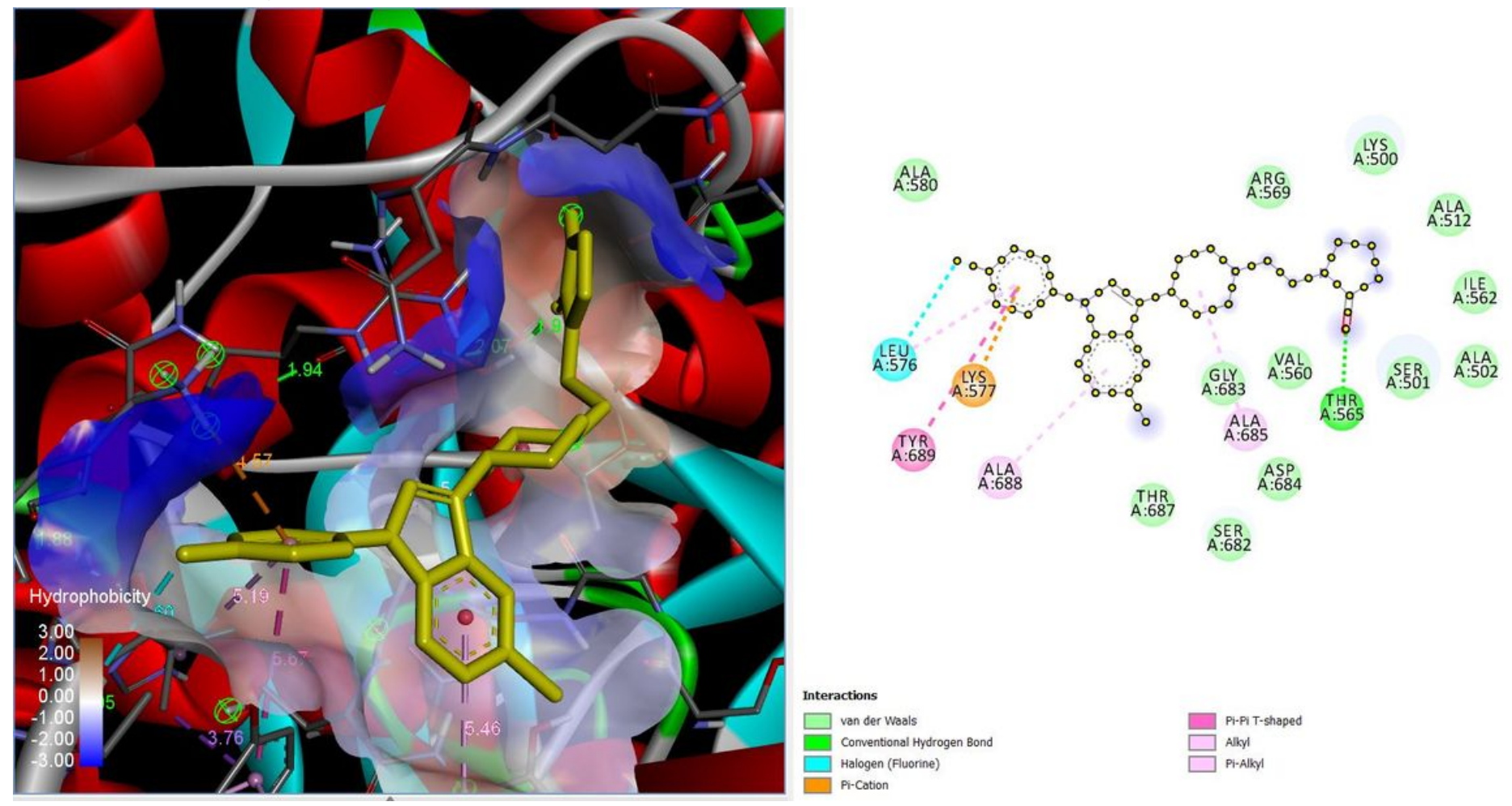

Figure 16

Nelfinavir in its docking space in RdRp visualized using Drug Discover Studio. 6 target amino acids were interacted by it.
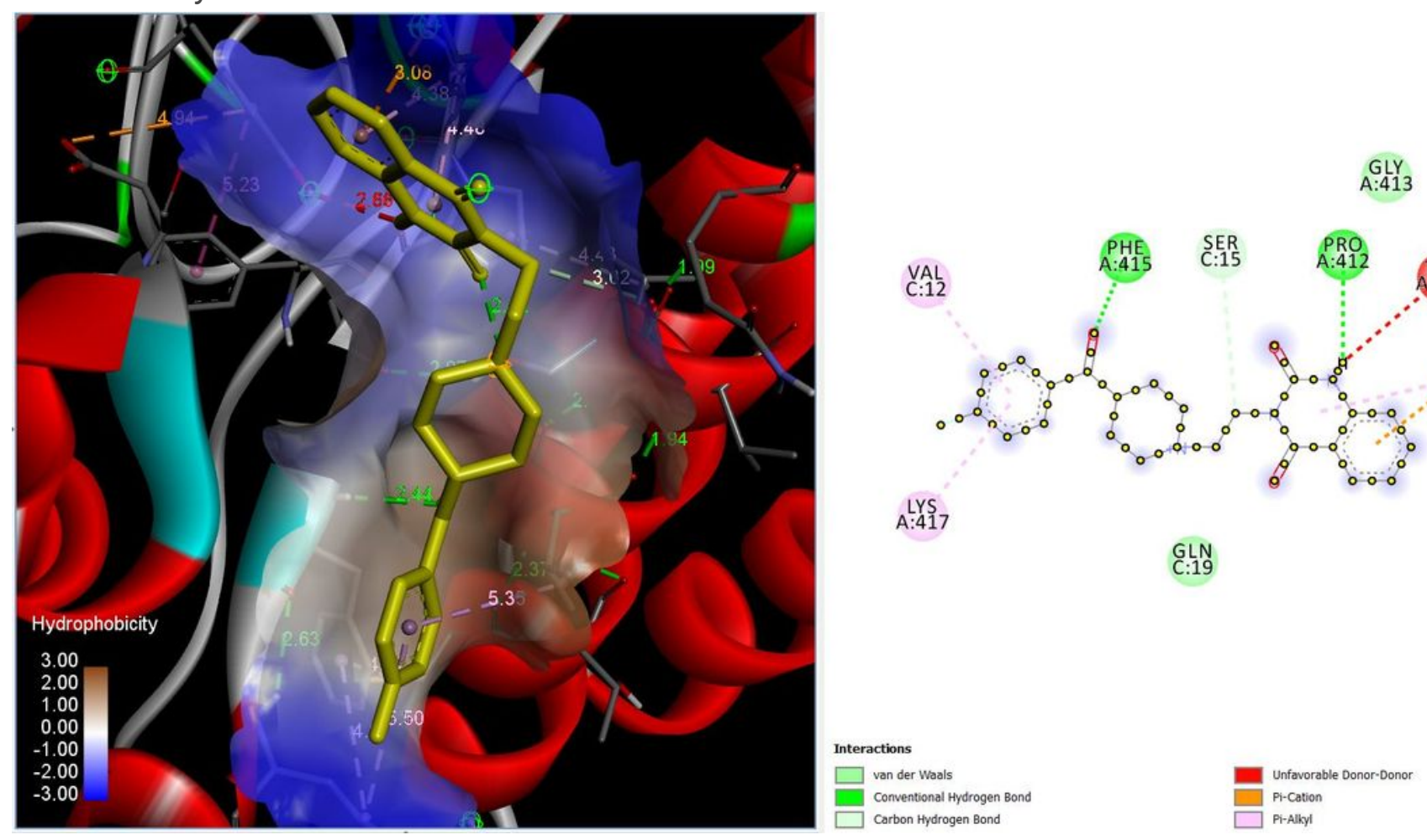

Figure 17 
Sitagliptin in its docking space in RdRp visualized using Drug Discover Studio. 4 target amino acids were interacted by it.

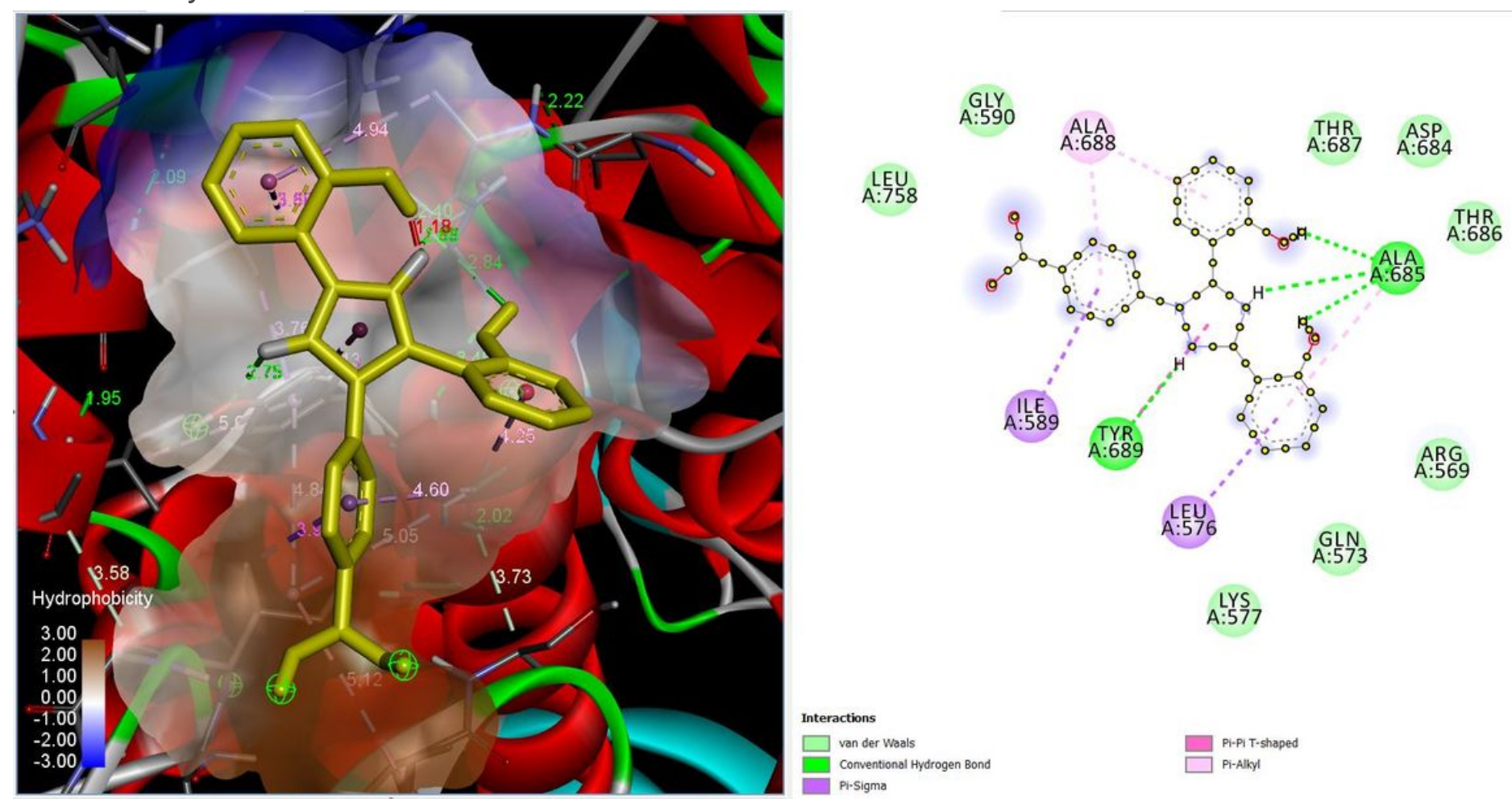

Figure 18

Sitaxentan in its docking space in RdRp visualized using Drug Discover Studio. 5 target amino acids were interacted by it.
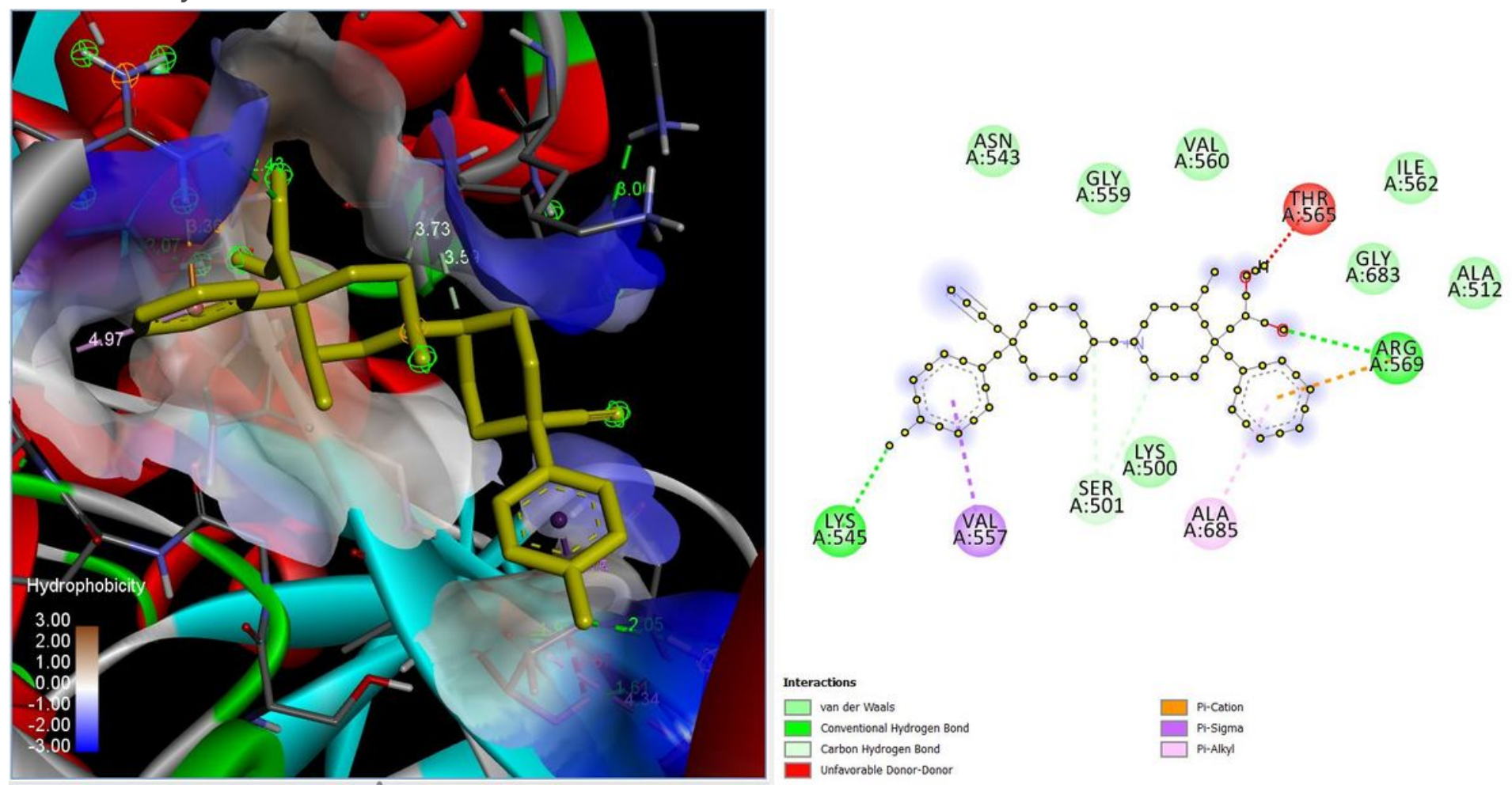

Figure 19 
5-methyltetrahydrofolate in its docking space in RdRp visualized using Drug Discover Studio. 5 target amino acids were interacted by it.
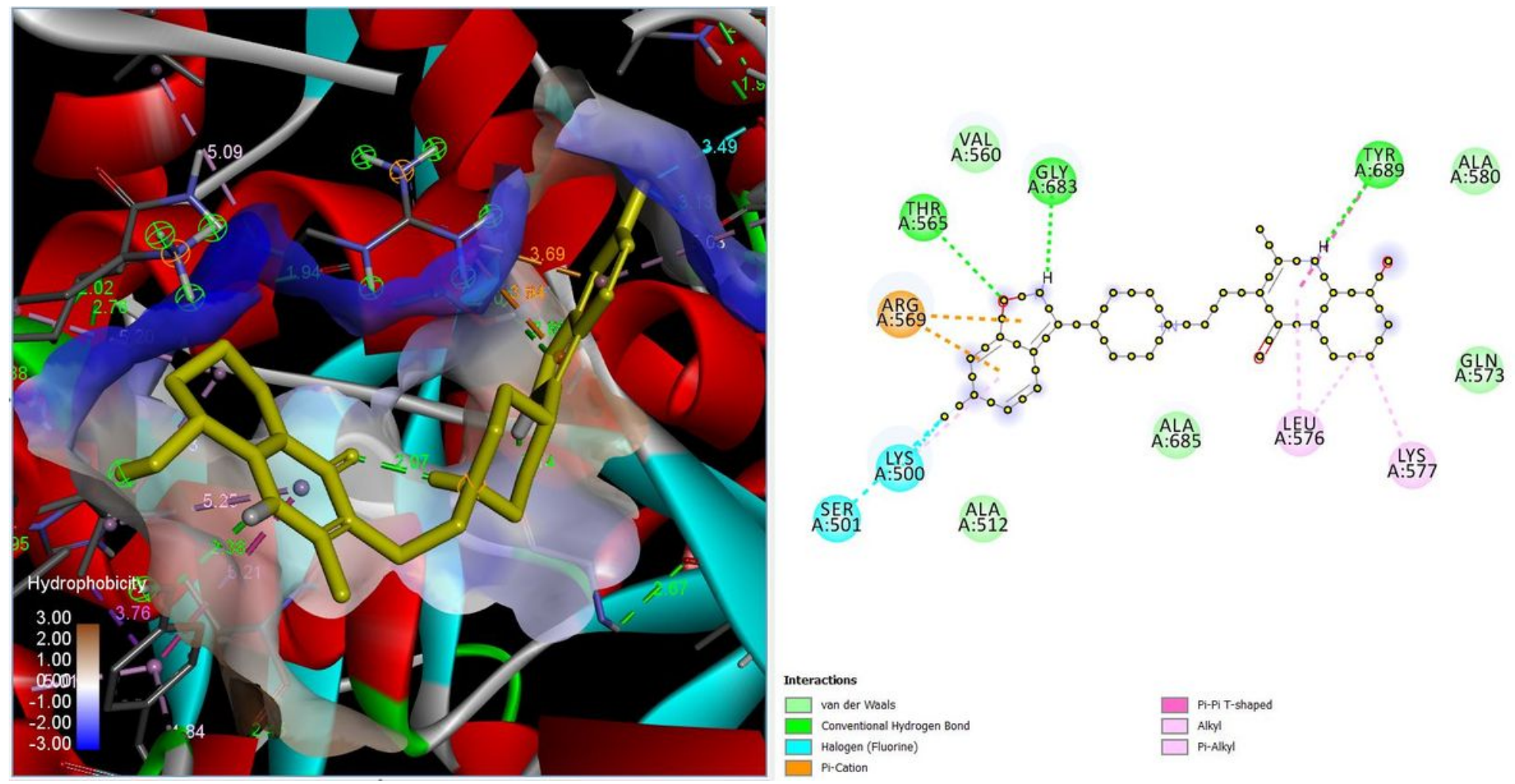

Figure 20

Levocabastin in its docking space in RdRp visualized using Drug Discover Studio. 3 target amino acids were interacted by it.
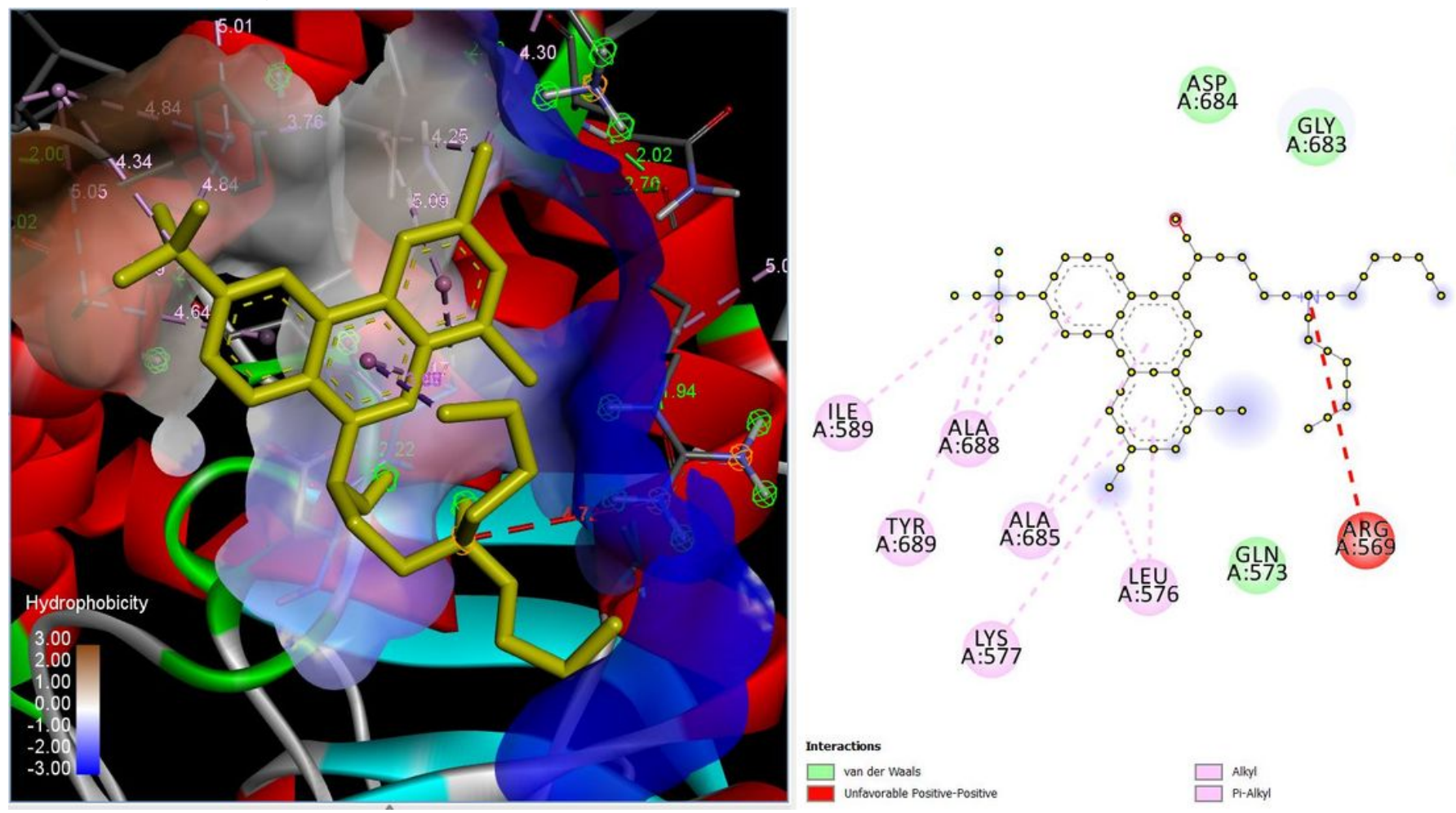

Figure 21 
Deferasirox in its docking space in RdRp visualized using Drug Discover Studio. 2 target amino acids were interacted by it. 